\title{
UC-NRLF
}

|||||||||| ||| ||||||||||||||||||||||

"घhim

B 3071489

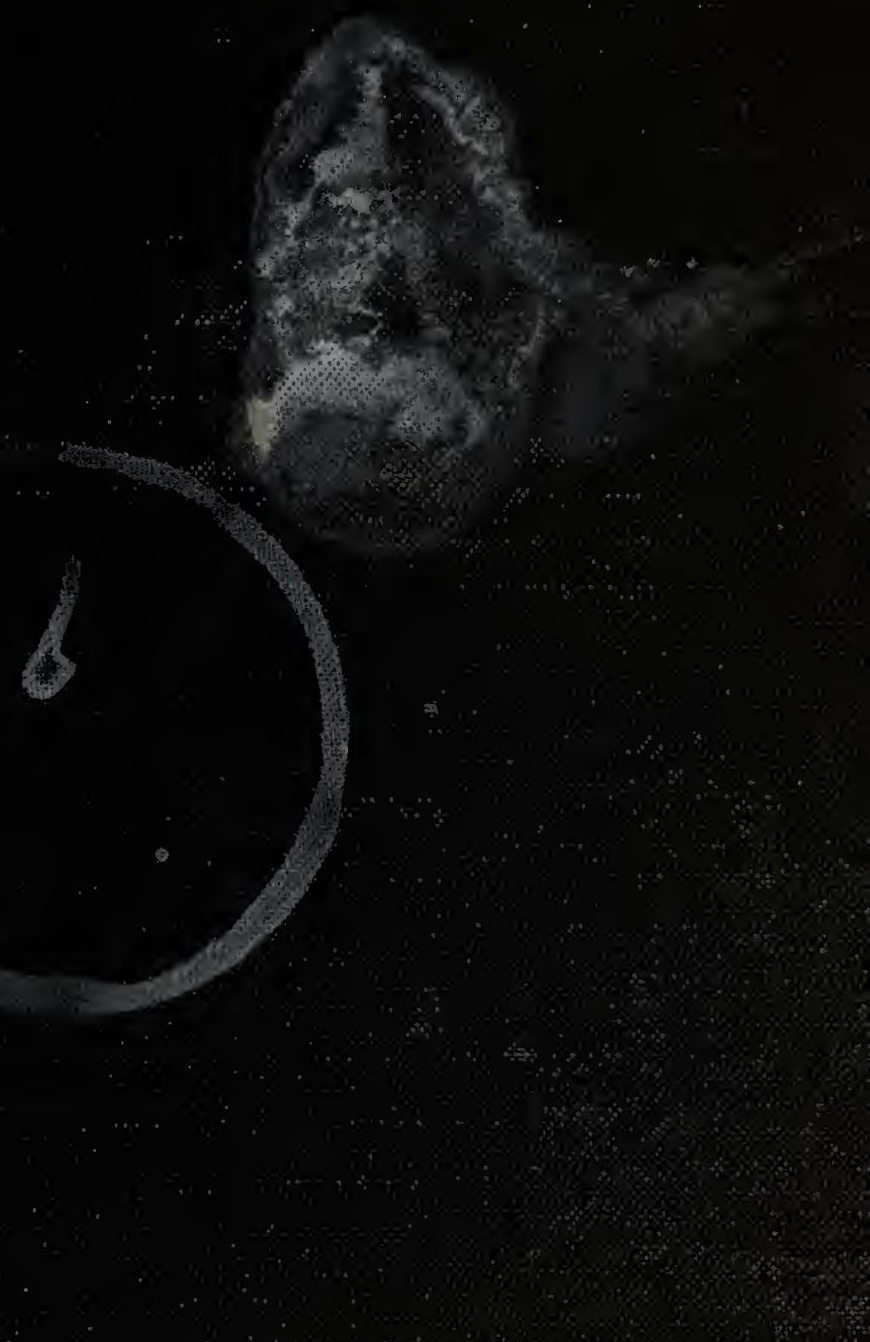




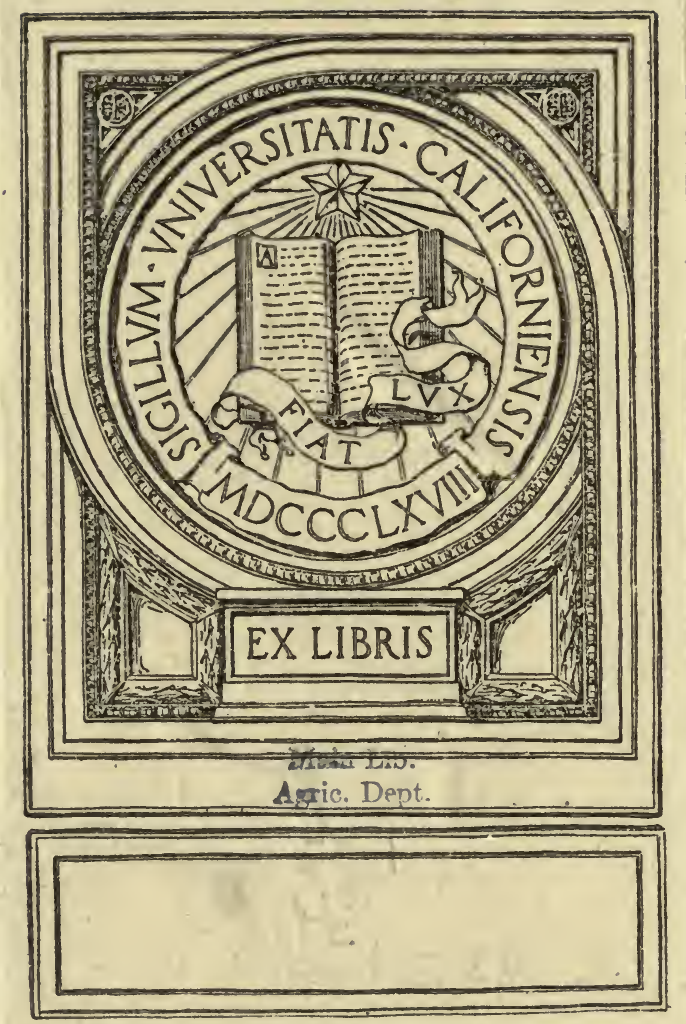


Digitized by the Internet Archive in 2007 with funding from Microsoft Corporation 


\title{
United States Department of Agriculture,
}

\author{
BUREAU OF CHEMISTRY-Circular No. 67.
}

H. W. WILEY, Chief of Bureau.

\section{A METHOD FOR THE DETERMINATION OF TIN IN CANNED FOODS.}

By Herman Schreiber and W. C. Taber, Assistant Chemists.

The determination of tin in canned foods, which has recently become of increased importance, may be made by several methods, each having its advantages and defects. Ashing the material at a very low heat in a muffle will give correct results with some foods under some circumstances, but not always, and when the composition of the material is not known, it is not safe to apply this method. Moreover, tin and its salts are volatilized by heat in the presence of chlorid of ammonia and probably with other chlorids also, since it is well known that sodium chlorid volatilizes on heating strongly. When small amounts of a metal are to be recovered from a large amount of organic matter, the danger of mechanical loss in the fumes is very great. After ashing, the ash must be fused with caustic alkali if all of the tin is to be recovered, so that this process does not offer any advantage over the method herein proposed in regard to the time necessary for making the determination.

Munson ${ }^{1}$ has proposed carbonizing the organic matter with sulphuric acid and heat, and ashing in a muffle with the aid of nitric acid. Determinations made by this method did not give satisfactory duplicates, and the results represented only about 50 per cent of the tin present, as determined by sulphuric acid digestion (Table 1) in Halenke's wet ashing method, as modified by Schryver. ${ }^{2}$ In the latter method the organic matter is destroyed by digestion with large amounts of sulphuric acid with the aid of potassium sulphate.

1 U. S. Dept. Agr., Bureau of Chemistry Bul. 107, Revised, p. 62.

2 Report 7 of the Local Government Board (medical department) of Great Britain on the presence of tin in certain canned goods.

$71511^{\circ}-$ Cir. $67-11$ 
- TABLE 1.-Comparison of the Munson metiod and the sulphuric acid or wet comoustion method (Schryver).

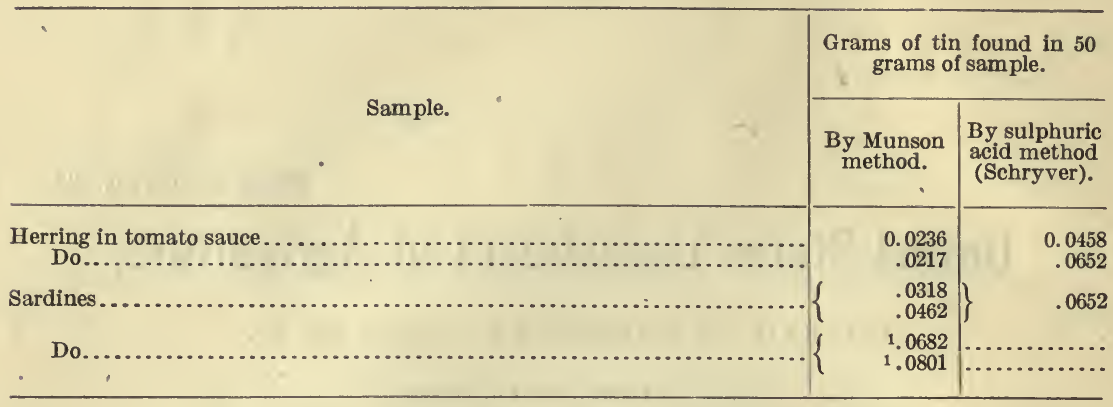

1 Wet combustion not made, given to show poor concordance of results.

Table 2 shows that the results obtained when the precipitates are weighed as stannic oxid $\left(\mathrm{SnO}_{2}\right)$ are as correct as when the precipitate is dissolved and determined electrolytically ${ }^{1}$ as tin. Schryver has shown that the wet combustion method gives accurate results and good duplicates. This is also shown by the determinations given in Table 2. However, this method has some very serious practical defects. When tin is to be determined in meat, fish, or sirups, only 25 grams of material can be digested in one flask. This necessitates making two digestions in order than an amount can be used which will avoid the great multiplication of the analytical error which would occur if the result were calculated as milligrams per kilogram. Using such small quantities of the sample increases the error of weighing and sampling, and, moreover, limits the amount of work which can be done under ordinary conditiors. The flasks have a tendency to break during the digestion, which, together with the foaming of the material, requires constant attention and considerable experience before satisfactory results can be obtained. No attempt was made to determine the amount of tin which could be recovered by the various methods by adding known amounts of soluble tin salts to food, since this would not give conditions analogous to those met in practice.

TABLE 2.-Comparison of results by wet combustion or sulphuric acid method and by electrolysis.

[Grams of tin per 50 grams of sample.]

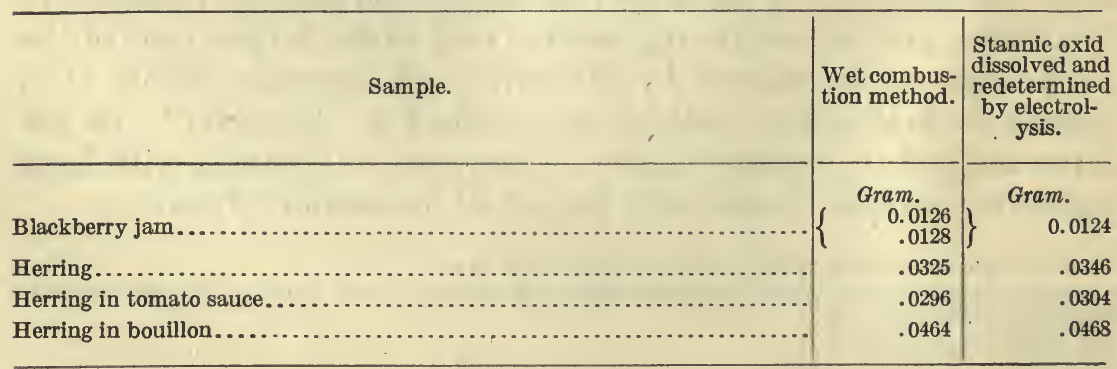


Our work indicated that the following was the most satisfactory method of making a sulphuric acid digestion: Weigh 25 grams of fish or meat or 50 grams of vegetables into a beaker, wash into an 800 or 1,000 c. c. Kjeldahl flask, add from 100 to 150 c. c. of water, 25 grams of potassium sulphate, 50 c. c. of sulphuric acid (sp. gr. 1.84), and a few glass beads. Place over a small flame, rotate a few times till it boils, then increase heat and boil vigorously until the water is boiled off and fumes of sulphur trioxid appear in the neck of the flask and settle back, remove it from the flame before it foams or caking will ensue and the flask break in subsequent boiling. Add 50 c. c. of sulphuric acid (sp.gr. 1.84) turn the flame down so that it just touches the flask, and heat gently until the mass boils quietly. Again increase the heat and boil vigorously until decolorized. If the flask is placed in an asbestos ring, cracking can often be avoided. "This digestion requires at least six hours.

To determine the accuracy of duplicate determinations by the sulphuric acid method, samples of canned fish were very carefully prepared by passing them through a sausage grinder and then thoroughly mixing them by hand. From these mixtures duplicate weighings of 25 grams were made on an analytical balance to \pm 0.05 gram and the following results were obtained:

TABLE 3.-Duplicate determinations of tin by the sulphuric acid or wet combustion method.

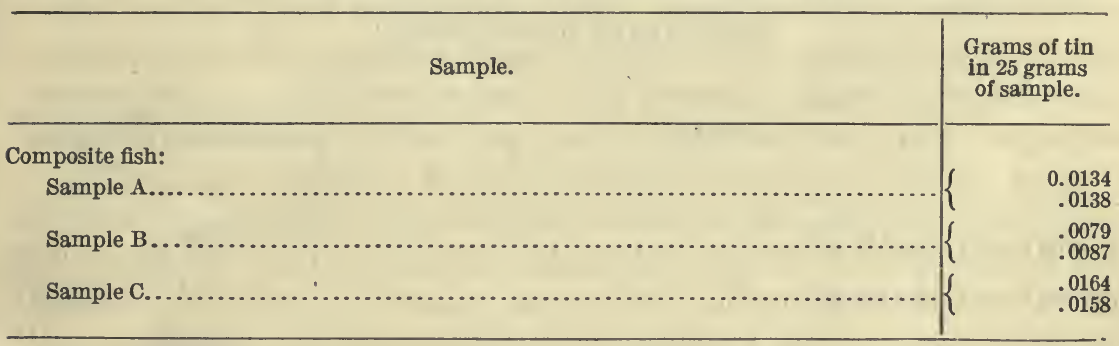

To determine the effect of the presence of sodium chlorid on results obtained by the wet combustion method, 10 grams of salt were added to the material to be analyzed. The figures given in the following table prove that this had no effect on the results:

TABLE 4.-Determination of tin by the sulphuric-acid method in the presence of salt.

[Grams of tin in 50 grams of sample.]

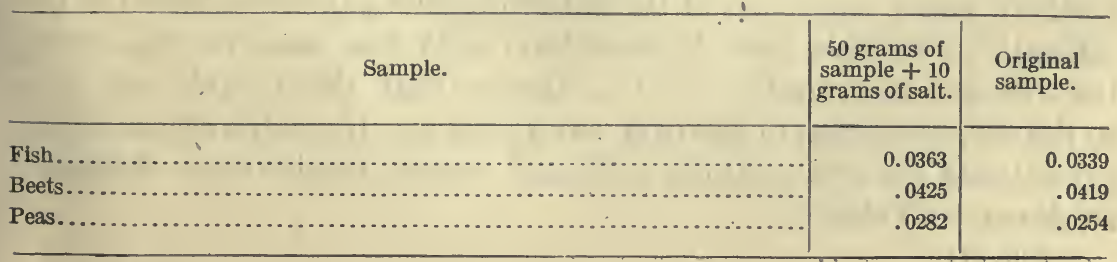


If tin is to be estimated in foods, such as fish, which contain so great an amount of salt as not to be edible before soaking, it is better to determine the tin by the sulphuric-acid method, as large amounts of salt would interfere with the burning off of the carbon by the proposed method. However, such cases wculd be very rare, as salt fish are commonly packed in wood and not in tin.

The method proposed on page 6 requires practically no experience in its manipulation, and a large amount of the sample is used, in both of which points it is superior to the wet combustion method. It was at first thought that the destruction of the organic matter could be effected by an alkaline fusion, using alkaline hydrate and nitrates. However, when material, such as fish, which contains fat, is to be analyzed, it is impossible to make such a fusion, as it will burn and blow out of the crucible. When alkaline hydrates are used alone, a solid cake results which retards the complete destruction of carbon by coating the small particles. A mixture of hydrate, carbonate, and magnesium oxid was found to give a very porous fusion, in which the carbon was easily oxidized in the muffle. The fusion was at first attempted in large nickel crucibles with very good results as shown by Table 5 .

TABLE 5.-Comparison of the sulphuric-acid or wet combustion method with alkali fusion in a nickel crucible by the proposed method.

[Grams of tin per 50 grams of sample.]

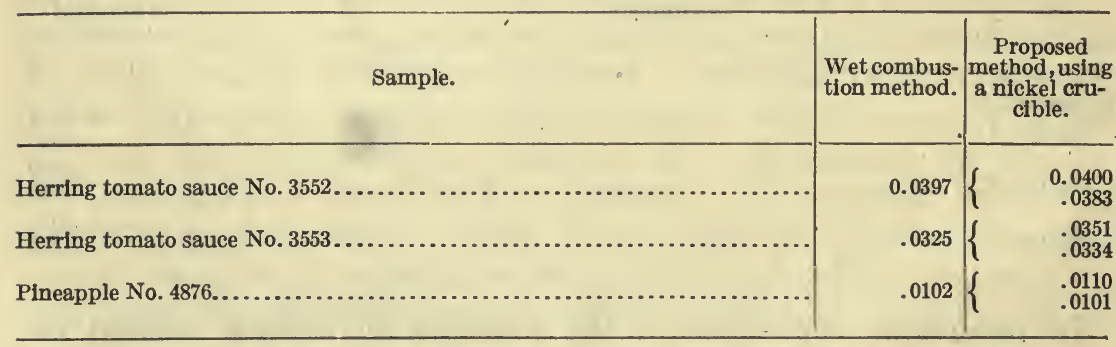

The nickel crucibles, however, were very seriously attacked, and since they are expensive, it was thought that fusion in an iron crucible would be a great advantage on account of the lower cost and the higher melting point of iron. When iron crucibles were first substituted, the results obtained were lower than by the wet combustion method, and a repetition of the determination gave no solution of the difficulty. At this time hydrochloric acid was used for dissolving the fusions and finally, on the theory that the trouble was due to the solvent action of the iron chlorid on the tin, sulphuric acid was substituted for hydrochloric acid and correct results were obtained, as shown in Table 6.

[Cir. 67] 
TABLE 6.-Comparison of results by the wet combustion method and the proposed method, using different solvents and an iron crucible.

[Grams of tin in $\mathbf{5 0}$ grams of sample.]

\begin{tabular}{|c|c|c|c|c|}
\hline & \multirow{2}{*}{ Sample. } & \multirow{2}{*}{$\begin{array}{l}\text { Wet com- } \\
\text { bustion } \\
\text { method. }\end{array}$} & \multicolumn{2}{|c|}{ Proposed method. } \\
\hline & & & $\begin{array}{l}\text { Using hy- } \\
\text { drochloric } \\
\text { acid. }\end{array}$ & $\begin{array}{l}\text { Using sulphu- } \\
\text { ric and nitric } \\
\text { acids. }\end{array}$ \\
\hline Beets.... & & 0.0419 & 0.0331 & $\left\{\begin{array}{r}0.0416 \\
.0403\end{array}\right\} 0.0410$ \\
\hline Tomatoes.. & & .0345 & .0290 & $\left\{\begin{array}{l}.0331 \\
.0310\end{array}\right\} .0320$ \\
\hline Peas... & & .0254 & .0252 & $\left\{\begin{array}{l}.0283 \\
.0268\end{array}\right\} .0276$ \\
\hline Corn... & & .0277 & .0238 & . $\ldots \ldots \ldots \ldots$ \\
\hline Apples. . & & .0338 & .0239 & $\left\{\begin{array}{r}.0320 \\
.0343\end{array}\right\} .0332$ \\
\hline Spinach.... & & .0048 & .0060 & .............. \\
\hline Mushrooms... & & .0107 & .0147 & .................. \\
\hline Canned fish... & & .0325 & & $\left\{\begin{array}{l}.0298 \\
.0328\end{array}\right\} .0313$ \\
\hline
\end{tabular}

The results reported in Tables 4 to 7 were obtained in the preliminary work done in developing the proposed method. In the method, as then used, 5 grams of magnesium oxid were employed, and the solution of the fusion was not concentrated until it fumed with sulphuric acid, but was simply boiled down, and blue ribbon paper was used for the first filtration. It was found that the fusions could be burned at a lower temperature if 10 grams of magnesium oxid were used, which makes the burning much easier and materially lengthens the life of the crucible. It was also found best to use a larger amount of acid, as specified in the method, since under certain circumstances all of the tin was not dissolved when only 50 c.c. of $1: 1$ sulphuric acid and 5 c.c. of nitric acid were used. The use of blue ribbon paper was also abandoned, since it decreased the speed of filtration, and, furthermore, it has no apparent advantage (Table 7) when the precipitation is made as specified in the proposed method. The experimental data indicate that sulphuric acid alone will not take all of the tin into solution, although no explanation of this can be offered.

[Cir. 67] 
TABLE 7.-Comparison of results by the wet combustion method and proposed method using blue ribbon filter papers and sulphuric acid.

[Grams of tin in 50 grams of sample.]

\begin{tabular}{|c|c|c|c|}
\hline & Sample. & $\begin{array}{l}\text { Wet com- } \\
\text { bustion } \\
\text { method. }\end{array}$ & $\begin{array}{l}\text { Proposed } \\
\text { method, using } \\
\text { blue ribbon fil- } \\
\text { ter papers } 1 \text { for } \\
\text { the first filtra- } \\
\text { tion and } 50 \mathrm{cc} \text {. } \\
\text { of } 1: 1 \text { sulphuric } \\
\text { acid and } 5 \mathrm{cc} \text {. of } \\
\text { nitric acid to dis- } \\
\text { solve the fusion. }\end{array}$ \\
\hline Beets. . & & 0.0419 & $\left\{\begin{array}{c}0.0403 \\
.0424\end{array}\right\} 0.0414$ \\
\hline Peas... & & .0254 & $\left\{\begin{array}{ll}.0265 \\
.0275\end{array}\right\} .0270$ \\
\hline Corn.... & & .0277 & $\left\{\begin{array}{c}.0269 \\
.0276\end{array}\right\} \cdot 0273$ \\
\hline Apples. & & .0338 & .0323 \\
\hline Fish.. & & .0325 & $\left\{\begin{array}{ll}.0303 \\
.0329\end{array}\right\} .0316$ \\
\hline
\end{tabular}

1 These tin oxids were only very slightly discolored, being very nearly the same shade as those from the wet combustion.

The proposed method has several advantages over the wet combustion method: (1) It decreases the errors of sampling; (2) it decreases the errors due to multiplication when calculating to milligrams per kilogram; (3) there need be no loss of samples if the material is properly ground and not heated too rapidly on the hot plate; 4 ) in the wet ashing methods it is necessary to neutralize a large amount of acid before precipitating with hydrogen sulphid.

Molasses and heavy sirups must be dried longer on the hot plate than any other material analyzed, over three hours being necessary to dry these properly. However, as it requires no attention at this stage, this is not a serious defect as compared with the difficulties of determining tin by the wet ashing method. In drying any material on the hot plate it is not necessary to dry it down to a hard crust if it is put in a cold muffle and heat applied gradually. If tin is to be determined in oils, less of the sample should be used, so that there will be an excess of free sodium hydrate. The sulphuric acid method for the determination of tin can not be applied to oils, and therefore no comparison of the two methods can be made.

\section{THE PROPOSED ALKALI FUSION METHOD.}

Pass the sample through a meat-grinding machine and mix the resulting mass well so as to get as homogeneous a sample as possible. Weigh 100 grams of the sample and 10 grams of magnesium oxid into an 8-ounce wrought-iron crucible on a rough balance. Add 50 c. c. of an aqueous solution containing 150 grams of sodium hydrate and 100 grams of sodium carbonate per liter, stir well with a short piece of stout glass rod, add 75 c.c. of 95 per centalcohol and stir again. Place on the steam bath and evaporate the alcohol. This must be done with care, stirring frequently at the beginning, else it may bump or foam over, but if the material is gradually heated there is no danger of loss. If the material foams, remove from the steam bath or hot plate for a moment,

[Cir. 67] 
and then replace on the bath and it will usually boil quietly. Large lumps in the mass will cause bumping; therefore the sample should be as fine as possible. Fish liquefy on the steam bath, boil down quietly, and without bumping. After danger from frothing has ceased, apply the full heat of the steam bath. This evaporation requires about one hour. Transfer to a hot plate covered with a thin sheet of asbestos and dry down gradually, running at from $130^{\circ}$ to $160^{\circ} \mathrm{C}$. at first (determine temperature by laying a thermometer on the asbestos); then raise the temperature and continue the boiling, finally using the full heat of the hot plate, which should be sufficient to boil off sulphuric acid. This requires from one and a half to two hours. Place in a cold muffle, heat gradually until all volatile matter is driven off, and then burn until all of the carbon is destroyed. This requires from two to three hours. Remove from the muffle as soon as burned, cool, cover the residue with water, and let stand a few minutes. Using an iron spatula, scrape and wash the contents of the crucible into a 600 c. c. beaker. Cover the beaker and add gradually 40 c. c. of dilute sulphuric acid $(1: 1)$ and $10 \mathrm{c}$. c. to the crucible containing some water. Rotate, scrape the sides of the crucible with a spatula, and wash into the beaker. When the peaction is ended, add 50 c. c. of sulphuric acid (sp. gr., 1.84) and 30 c. c. of nitric acid (sp. gr., 1.42). Cover with a watch glass and boil briskly on a hot plate, finally with the full heat of the hot plate, until the residue gives off fumes of $\mathrm{SO}_{3}$. Allow to fume for 10 minutes, remove from the hot plate and allow to cool but not to solidify. Pass the stem of a funnel bent at an angle over the lip of the beaker and under the cover glass and add successively small amounts of distilled water through the funnel from a wash bottle until violent action has ceased. Then add rapidly about 150 to 200 c. c. more water through the funnel and remove the funnel and watch glass, washing into the beaker with distilled water. Stir the cake in the beaker and wash into a 1-liter erlenmeyer with distilled water.

The total volume in the erlenmeyer at this time should be about 300 to $400 \mathrm{c}$. c. Cool, pass in hydrogen sulphid for a few seconds, rotate the flask and add 28 per cent ammonium hydroxid slowly until the black color of the precipitated iron sulphid just persists on rotating the flask. Immediately make acid with $1: 1$ sulphuric acid and add 10 c. c. excess of the dilute acid. Dilute the contents of the flask to 1 liter with boiling water and continue passing in a rapid stream of hydrogen sulphid for 25 minutes more, cork, and let stand over night. The next morning heat on the steam bath for about half an hour, rotating the flask two or three times during the heating, partly cool by setting in cold water, so that the flask can be handled easily, and filter onto a $12.5 \mathrm{~cm}$. ashless white ribbon paper, No. 589, washing the filter with a solution consisting of $50 \mathrm{c}$. c. of glacial acetic acid and $100 \mathrm{c}$. c. of a saturated solution of ammonium acetate, made up to a liter with distilled water. Wash the precipitate six times with this solution, filling the filter at each washing. Return the filter paper and precipitate to the erlenmeyer, add 100 c. c. of 20 per cent potassium hydroxid and boil over a free flame for a couple of minutes until the filter paper is broken up and the solution is clear. (The flask can be manipulated over the flame easily with a large wooden test-tube holder made of strips of pine wood and rubber bands.) Immediately decant through a double white ribbon filter paper of $12.5 \mathrm{~cm}$. into a 400 c. c. beaker, washing the flask and filter with successive portions of hot water until the filtrate comes through colorless. The filtrate will have a volume of about 200 to 300 c. c. Add 20 c. c. of concentrated hydrochloric acid to this solution, stir, add a few drops of phenolphthalein and add concentrated hydrochloric acid from a burette until the dark color of the solution disappears, then add 1 c. c. excess of the acid. Test with a strip of litmus and see that the solution is acid, stir well, place on a steam bath, heat for 20 minutes, cover, and let stand over night.

In the morning test the supernatant liquor, which should be perfectly clear and brilliant, with a piece of blve litmus. If not acid, make so with concentrated hydrochloric acid and then add an excess of 1 c. c. If the supernatant liquor is acid and

[Cir. 67] 
turbid, make alkaline with potassium hydroxid and then acid with 1 c. c. excess. The solution must neither be alkaline nor too acid, or there will be trouble in filtering and washing. Heat on a steam bath for half an hour, stirring two or three times. Filter onto a $12.5 \mathrm{~cm}$. white ribbon paper. The precipitate will sometimes run through, and must be returned until the filtrate is perfectly clear and brilliant. If the solution to be filtered is stirred vigorously, allowed to stand until the precipitateclots, and then poured onto the filter, refiltering may usually be avoided. The filtrate must be perfectly clear or some tin will be lost. Wash alternately with distilled water and the ammonium acetate solution previously mentioned until the filtrate obtained from a washing with distilled water is free from chlorids. (Do not mistake the precipitate given by the acetate solution and silver nitrate, which is soluble in water, for silver chlorid.) This requires washing until the volume of the filtrate is $200 \mathrm{c}$. c. or more. Fill the filter at each washing. Place the moist filter in a porcelain crucible, dry and char on an asbestos gauze, and burn off all carbon over the free flame. Cool in a desiccator and weigh as stannic oxid.

Table 8 gives a comparison of the results obtained by this method and those obtained by Schryver's method.

TABLE 8.-Comparison of proposed method with Schryver's sulphuric-acid method.

[Grams of tin in 100 grams of sample.]

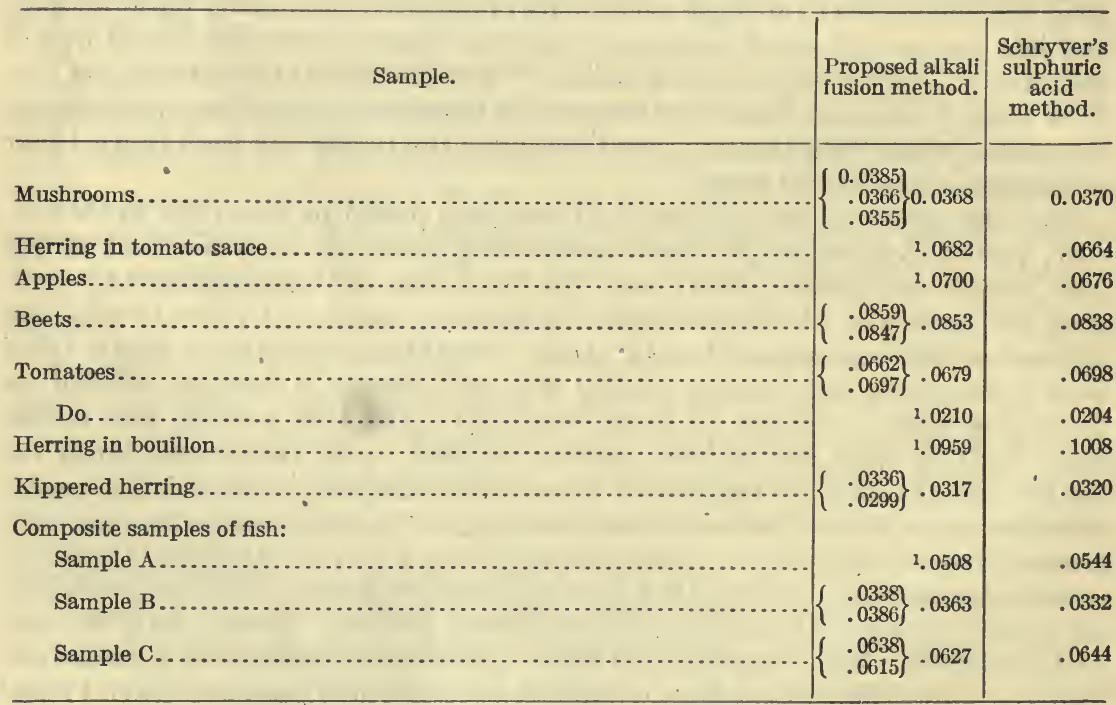

1 Duplicates not run, as there was not sufficient material.

Experiments were made to determine the relative value of potassium hydrate and ammonium sulphid as solvents for tin sulphid and it was found that they gave concordant results. In one instance the solution by potassium hydrate gave 0.0313 gram of stannic oxid, and the ammonium sulphid gave 0.0318 gram. The sample of ketchup on which these determinations were made contained a large amount of sand, and the results show that there is no danger of silica contaminating the stannic oxid when potassium hydroxid is used. 
Copper was found in some of the foods examined, and since copper sulphid is more soluble in ammonium sulphid than in potassium hydrate, it seemed advisable to use the latter.

A muffle 9 by 21 inches will hold 10 crucibles at a time, and one analyst can easily make 20 determinations in a week. If the heating in the muffle is gradual and the crucibles are removed as soon as all of the carbon is destroyed, the crucible will not be blistered on the outside and only slightly on the inside, and can be used for repeated fusions. If the crucibles have been spun with a copper or brass tool, the traces of copper adhering to the crucible should be filed off. Fifteen determinations can be weighed, dried, and burned in nine hours, using a hot plate 14 by 18 inches, and a muffle 9 by 21 inches. This requires three hours' continuous watching, and then only an occasional inspection of the muffle and changing the position of the crucibles. Straight ashing, on the other hand, requires at least seven hours' burning in the muffle if even approximately accurate results are to be obtained and if the food can be put into the muffle without previous drying.

Two hundred and fifty determinations were made in working out the details of this proposed method.

[Cir. 67] 
RETURN

MAIN CIRCULATION

TO

ALL BOOKS ARE SUBJECT TO RECALL RENEW BOOKS BY CALLING $\underline{\text { 642-3405 }}$

\section{DUE AS STAMPED BELOW}

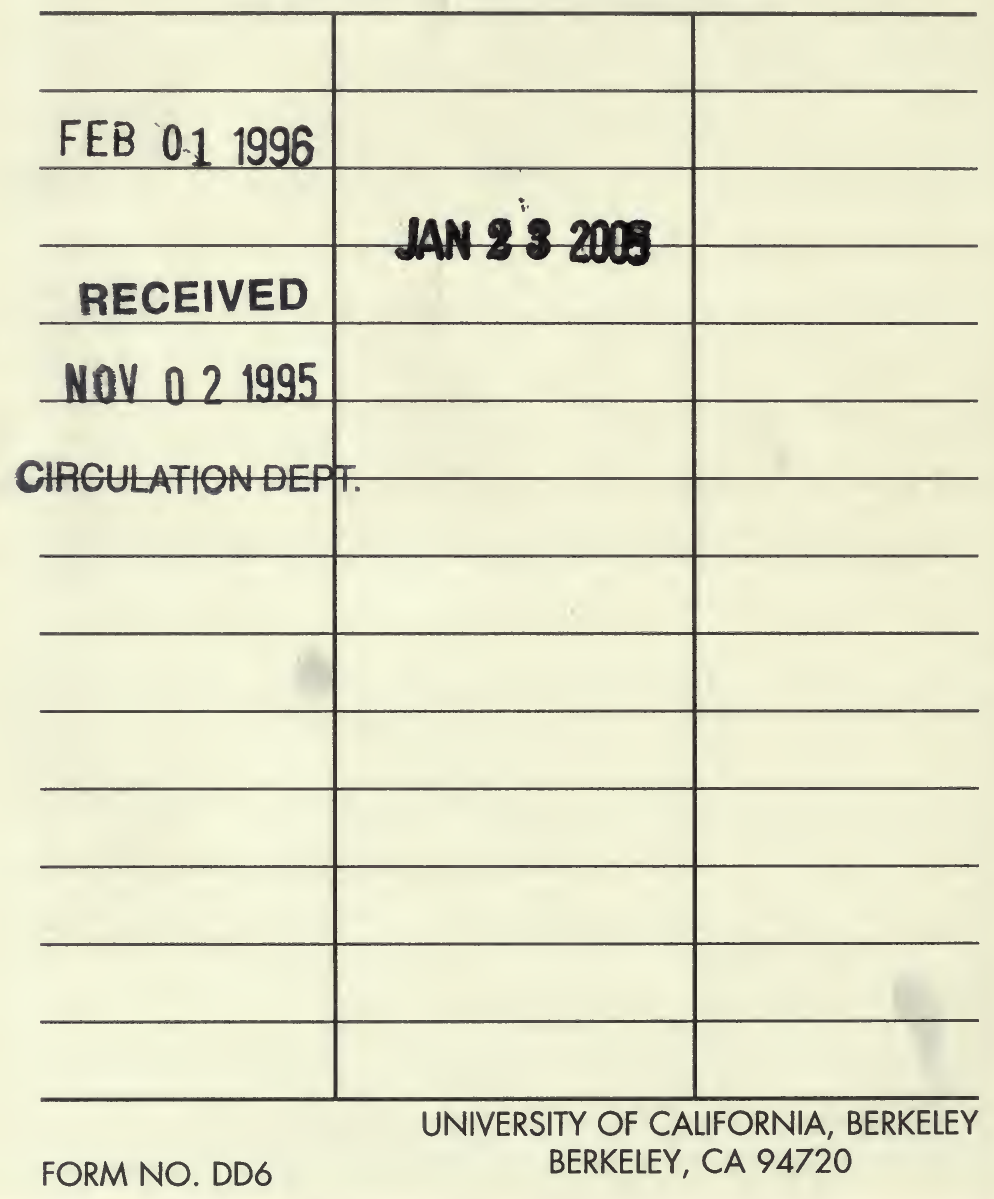




\section{YC 6940?}

$$
\text { r.ten }
$$

U. C. BERKELEY LIBRARIES

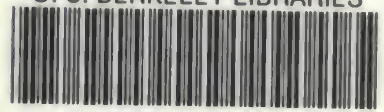

C051402935 
(3)

s.

$\alpha$

(6)

is.

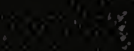

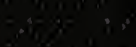

